\title{
A Unified MAC Layer Framework for Ad-hoc Networks with Smart Antennas *
}

\author{
Karthikeyan Sundaresan and Raghupathy Sivakumar \\ School of Electrical and Computer Engineering \\ Georgia Institute of Technology \\ $\{$ sk,siva\}@ece.gatech.edu
}

\begin{abstract}
Smart antennas represent a broad variety of antennas that differ in their performance and transceiver complexity. The superior capabilities of smart antennas, however, can be leveraged only through appropriately designed higher layer network protocols, including at the medium access control (MAC) layer. Although several related works have considered such tailored protocols, they do so in the context of specific antenna technologies. In this paper, we explore the possibility for a unified approach to medium access control in ad-hoc networks with smart antennas. We first present a unified representation of the PHY layer capabilities of the different types of smart antennas, and their relevance to MAC layer design. We then define a unified MAC problem formulation, and derive unified MAC algorithms from the formulation. Finally, using the algorithms developed, we investigate the relative performance trade-offs of the different technologies under varying network conditions.
\end{abstract}

\section{Categories and Subject Descriptors}

C.2.2 [Computer-Communication Networks]: Network Protocols

\section{General Terms}

Algorithms, Design, Performance

\section{Keywords}

Ad-hoc networks, MAC layer, Smart antennas

\section{INTRODUCTION}

Smart antennas represent a broad variety of antennas that differ in their performance and transceiver complexity, including switchedbeam antennas, steered-beam antennas, adaptive array antennas, and multiple-input-multiple-output links. A switched-beam antenna has a pre-determined antenna array pattern that can be pointed to any of a small number of directions. The ability of such antennas to concentrate power in a certain direction provides a directional gain.

\footnotetext{
* This work was funded in part by the National Science Foundation under grant CCR-0313005.
}

\footnotetext{
Permission to make digital or hard copies of all or part of this work for personal or classroom use is granted without fee provided that copies are not made or distributed for profit or commercial advantage and that copies bear this notice and the full citation on the first page. To copy otherwise, to republish, to post on servers or to redistribute to lists, requires prior specific permission and/or a fee.

MobiHoc'04, May 24-26, 2004, Roppongi, Japan.

Copyright 2004 ACM 1-58113-849-0/04/0005 ...\$5.00.
}

Steered-beam antennas also have pre-determined patterns, but they can be pointed to any of a near-continuous set of directions. While steered-beam antennas are optimal in terms of signal-to-noise ratio (SNR) in free space with no interference, their performance deteriorates in a multipath environment where multiple copies of the signal can arrive from different directions [1]. An adaptive array receiver constructively combines the copies, yielding array gain, which is the factor increase in the average SNR equal to the number of antennas [2]. If the antennas are sufficiently far apart, then the likelihood of the deepest fades is decreased, corresponding to diversity gain. A transmit digital adaptive array (DAA) can also provide array and diversity gains, augmenting those of a receiver array. Recently, the use of DAAs at both ends of the communication link has gained consideration, resulting in a technology popularly referred to as the multiple input multiple output (MIMO) technology. The presence of multiple elements at both ends of the link creates independent channels in the presence of multipath or rich scattering. Multiple independent data streams can be transmitted simultaneously (also known as spatial multiplexing) on these different channels to provide extremely high spectral efficiencies [3].

The superior transmission, reception, and interference suppression capabilities of smart antennas have inspired the consideration of their use in wireless ad-hoc networks that are inherently interference limited. However, the capabilities of the antennas can be effectively leveraged only through appropriate changes to higher layer network protocols. Hence, in recent years, several related works have investigated the question: What changes need to be made at the medium access control (MAC) layer and above in order to leverage the unique capabilities of smart antenna technologies? [4-6]. However, a common characteristic of such related works is that they focus on changes to higher layer protocols with respect to a single antenna technology.

Despite the specific differences in the characteristics of the antenna technologies, the fact that they still belong to the general umbrella of smart antenna technologies leads to an interesting question: Can the different smart antenna technologies be represented in any unified form? Or consequently, can unified algorithmic frameworks be developed for ad-hoc networks with smart antennas in general? With this question as the basis, in this paper we explore the problem of a unified approach to medium access control (MAC) in ad-hoc networks with smart antennas. Such an endeavor has the following benefits. First, a unified representation of the physical layer capabilities of the different types of smart antennas can help researchers see the relative merits of the technologies from the perspective of higher layer protocol design. Second, a unified problem formulation, and subsequent derivation of unified algorithms will enable specific aspects of the solutions developed for one class of antennas to be re-used for other classes as long as there are similar sub-problems. Finally, a unified MAC framework for the differ- 
ent classes of smart antennas will provide a very good platform for studying their relative performance trade-offs for varying network conditions.

In this context, we make the following contributions toward developing a unified MAC layer framework for ad-hoc networks with different types of smart antennas including omni-directional antennas, switched-beam antennas, adaptive array antennas, and MIMO links: (i) For the different antenna technologies, we identify the physical layer capabilities, their relevance to the MAC layer design, and MAC layer design considerations specific to the physical layer capabilities, and capture them through a unified representation; (ii) We provide a unified formulation of the problem of medium access control in ad-hoc networks with smart antennas, and show how the unified problem formulation can be applied to derive the specific formulation for a given technology; (iii) We derive unified centralized algorithms based on the above problem formulation; (iv) Finally, using the proposed algorithms we investigate the relative performance trade-offs of the different technologies, and identify key insights into how the technologies compare under varying conditions.

The rest of the paper is organized as follows: Section 2 provides some physical layer background. Section 3 discusses the MAC layer optimization considerations. Section 4 presents the problem formulation. Section 5 describes the unified (centralized) MAC framework. The relative performance of the antenna technologies are compared and evaluated in Section 6. Finally, Section 7 discusses related work, and conclusions are presented in Section 8.

\section{PHY LAYER BACKGROUND}

\subsection{Switched Beam Antennas}

Switched beam antennas employ multiple element arrays (MEAs) at both ends of the link. In any MEA, the signal that is sent to each of the antenna elements is weighted in both magnitude and phase before being transmitted. The specific set of weights that are applied to the different antenna elements is responsible for the antenna (radiation) pattern formed. In the case of switched beam antennas, a predetermined set of weights is used, each of which results in a beam pointing to a particular direction with a high gain. Since the weights are not required to be adapted, the complexity of the transceiver design is not significant. Note that the simplicity of not adapting the weights also comes with a limitation when these antennas operate in multi-path environments. Since the radiation pattern is fixed, when signals arrive with a large angular spread ( $>$ beam width) due to multi-path scattering this leads to a degradation in performance.

\subsection{Fully Adaptive Array Antennas}

While the switched beam antennas use a pre-determined set of weights for the antenna elements, the fully adaptive array antennas can adapt their weights so as to maximize the resulting SNR. This helps them cope with multi-path by adaptively changing their radiation pattern to accommodate the scattering resulting from multipath. However, this requires some feedback mechanism to help adjust the weights. As a result the signal processing complexity involved is more than that of a switched beam antenna. In addition to maximizing the gain for the desired signal, these antennas can also adaptively null interference. A $K$ element antenna is said to possess $K$ degrees of freedom ( $D O F S$ ), wherein it can adaptively null $K-1$ interferers completely even when they are uncorrelated with each other and with the desired signal. The maximization of the average SNR can be done by maximal ratio combining ${ }^{1}$ (MRC). The maximal ratio

\footnotetext{
${ }^{1}$ A PHY layer algorithm (mechanism) that determines the weights that maximize the SNR.
}

combining is generally done at the receiver, where the channel conditions can be estimated. However, for the transmitter to also help in the maximization of SNR, it necessitates some apriori knowledge of channel conditions at the transmitter too.

\subsection{MIMO Links}

A MIMO link employs digital adaptive arrays (DAAs) at both ends of the link. In the simplest configuration, the incoming data is demultiplexed into $K$ streams, and each stream is transmitted out of a different antenna with equal power, at the same frequency, with the same modulation format, and in the same time slot ${ }^{2}$. This is popularly referred to as spatial multiplexing. In fact, the above spatial multiplexing approach is optimal in terms of capacity when the transmitter array has no channel state information (CSI) [3]; hence the approach is often referred to as open-loop MIMO (OL-MIMO). At the receiver array, each antenna receives a superposition of all of the transmitted data streams. However, each stream generally has a different "spatial signature", and these differences are exploited by the receiver signal processor to separate the streams. On the other hand, when dependent streams are transmitted on the different elements, then it amounts to providing diversity, the benefits of which we explain in the subsequent section.

Another degree of classification of MIMO links is based on whether or not the transmitter uses CSI with respect to the receiver. If CSI is used at the transmitter, the MIMO link is referred to as closedloop MIMO (CL-MIMO). CL-MIMO is known to outperform OLMIMO under conditions of low SNR, correlated fading, and interference [7]. However, the CL-MIMO schemes require sophisticated signal processing capabilities at both the receiver as well as transmitter.

\section{CROSS-LAYER CONSIDERATIONS}

In this section, we first describe, for each of the antenna technologies, key PHY layer characteristics that are relevant to the MAC layer design. We present the details in terms of the communication pattern, potential gains and interference suppression capabilities. We then highlight key optimization goals that need to be accounted for in the MAC layer design in terms of leveraging gains, resource allocation and utilization, and scheduling. The problem formulation and centralized algorithms presented in the later sections, accommodate these optimization considerations.

\subsection{Switched Beam Antennas}

\subsubsection{Relevant PHY layer characteristics}

Communication pattern: Switched beam antennas rely on directionality for their communication, where the transmitter and the receiver are capable of forming directional beams towards each other.

Potential gains: The ability of the switched beam antennas to concentrate energy in a particular direction, provides an increase in SNR with respect to the desired signal resulting in a directional gain $G_{d}$. This gain can be bounded by,

$$
G_{d}=K^{2}
$$

in the case where both the transmitter and receiver knowing the direction of transmission to each other [1]. $K$ represents the number of elements at either of the ends (assumed to be the same at both ends for simplicity) and $p$ represents the path loss component. However, the above equation is true only for an LOS environment. In the case of multipath environments which are characterized by rich scat-

\footnotetext{
${ }^{2}$ Number of streams is actually determined by the rank of the channel matrix which in general is $\min (K$, multipath components).
} 
tering, the bound on the gain is determined by the scattering angle ${ }^{3}$ as the number of elements at both the ends increases [1]. Now, if the number of elements at both the ends of the link is assumed to be $K$, and if the scattering angles at the transmitter and receiver are $\alpha$ and $\beta$ respectively, then

$$
\begin{aligned}
G_{d} & =G_{t} G_{r}, \text { where } \\
G_{t} & =K, \text { if } \frac{360}{\alpha} \geq K \\
& =\frac{360}{\alpha}, \text { if } \frac{360}{\alpha}<K \\
G_{r} & =K, \text { if } \frac{360}{\beta} \geq K \\
& =\frac{360}{\beta}, \text { if } \frac{360}{\beta}<K
\end{aligned}
$$

The scattering angle saturates the gain beyond a certain number of elements because for higher number of elements, the antenna gain resulting from the increased elements is compensated by the loss of energy outside the main beam but within the scattering angle. Unlike in cellular environments, where the higher elevation of the base station helps keep the scattering angle low, ad-hoc networks - especially in an indoor setting - will be characterized by large scattering angles [1].

Interference suppression: These antennas do not provide flexible interference suppression owing to the use of pre-determined beam patterns. While they are considered to suppress interference along the non-active beams, the presence of side lobes contributes to accumulation of noise along these non-active beam directions and hence brings the down the SNR of the desired signal.

\subsubsection{MAC layer considerations}

Leveraging gains: The ability to focus energy in specific directions increases the spatial reuse in the network, thereby increasing the number of simultaneous parallel transmissions. We know that the directional gain represents the increase in SNR over that of an omni-directional element. But how does this gain help improve the network performance? The directional gain can be used in one of two ways ${ }^{4}$ : (i) for a given probability of error on the link, the gain can be used to increase the range of transmission/reception. This would in turn reduce the number of hops for multi-hop flows and hence increase the throughput. However, the interference range of a single transmission will also be increased, and the degree of spatial reuse in the network thereby reduced. The range extension factor $\left(R_{f}\right)$ can be bounded by ([1]),

$$
R_{f}=\left(G_{t} G_{r}\right)^{\frac{1}{p}}
$$

and (ii) the gain can be used to increase the capacity of the link (with no obvious negative side-effect). We know that the basic Shannon's upper bound on channel capacity (normalized to bandwidth) is given by

$$
C=\log _{2}(1+\rho)
$$

Now, when the average $\operatorname{SNR}(\rho)$ is increased by a factor $G_{d}$, there is a relative logarithmic increase in capacity $\left(C_{f}\right)$ to $C^{\prime}$ which can

\footnotetext{
${ }^{3}$ Angular spread within which significant energy of the transmitted signal can be received when it arrives in the form of multiple reflected components.

${ }^{4} \mathrm{~A}$ third and orthogonal possibility is to help minimize power consumption. However, we assume constant power in our discussions and hence do not focus on this possibility.
}

be approximated as,

$$
C_{f}=\frac{C^{\prime}}{C} \approx 1+\frac{\log _{2} G_{d}}{\log _{2} \rho}
$$

This increase in turn is achieved by means of using higher modulation rates for transmission/reception, while providing the same probability of error. In effect, more bits are transmitted per symbol (than in the case without the directional gain) while ensuring the same probability of error on the link.

Resource allocation and utilization: Every node has a single resource (for transmission) which simplifies resource allocation and utilization to the binary decision of gaining access to the channel or not.

Scheduling: The availability of a single resource eliminates the necessity to perform intelligent scheduling of links to leverage the advantages of location based contention prevalent in shared wireless channels [8].

\subsection{Fully Adaptive Array Antennas}

\subsubsection{Relevant PHY layer characteristics}

Communication pattern: As the name suggests, these antennas are capable of adapting their beam pattern to maximize the SNR of the desired signal. Hence, the notion shifts from directionality to availability of resources for communication. Each transmitter with $K$ elements, uses a single resource to transmit the signal, while using the remaining $K-1$ resources (DOFs) for suppressing other transmissions in its neighborhood.

Potential gains: The maximization of SNR achieved through the use of MRC at the receiver results in an array gain. Assuming that CSI is available at both the transmitter and receiver, the resulting array gain $G_{a}$ can be bounded by ( [9]),

$$
G_{a}=M N
$$

where $\mathrm{M}$ and $\mathrm{N}$ are the number of antennas at the transmitter and receiver respectively. While the gain from adaptive array antennas does not degrade with an increase in the degree of multipath (scattering angle) unlike switched beam antennas, yet, when the angular spreads are significantly large at the transmitter and receiver, the low correlation existing between the different signal components bounds the gain as ( [9]),

$$
G_{a}=(\sqrt{M}+\sqrt{N})^{2}
$$

In the case when all the DOFs are not used towards suppressing interference, then the remaining DOFs can be used to provide diversity gain. The diversity gain reduces the probability of the signal experiencing deep fades. While the array gain relates to the increase in the mean of the SNR at the output of the combiner, the diversity gain relates to the reduction in the variance of the SNR at the output of the combiner, relative to its variance prior to combining. The reduction in variance depends on the diversity order, which in turn depends on the degree to which the multipath fading on the different antenna elements is uncorrelated. The maximum diversity order afforded by a link with $\mathrm{M}$ transmit antennas and $\mathrm{N}$ receive antennas is $\mathrm{MN}$. While array gain continues to grow as more antennas are added, diversity gain tends to saturate [2].

Interference suppression: Adaptive array antennas are capable of flexible interference suppression. While the number of DOFs (resources) required for suppressing interference depends predominantly on the number of interfering streams transmitted, the strength of the interference and their spatial correlation also plays a notable role. Weak and highly correlated interference signals may not require as many DOFs to be sacrificed for suppression as the strong ones, thereby helping the node potentially accommodate more transmissions in its neighborhood. 


\subsubsection{MAC layer considerations}

Leveraging gains: As in the case of switched beam antennas, the array and diversity gains of adaptive arrays can be used toward range extension or capacity increase. However, if the resources at a node are all used up in suppressing interference, then we can consider array gain to be the dominant contributor, in which case, we obtain the bound on range extension as [1],

$$
R_{f}=\left(G_{a}\right)^{\frac{1}{p}}
$$

Since the diversity gain deals with the variance in SNR (unlike the array gain that deals with the mean SNR), the relation between diversity gain and number of elements is not direct. However, a measure of diversity, namely the diversity order can be directly related to the number of elements. On the other hand, if capacity increase is desired, then the average $\operatorname{SNR}(\rho)$ can be increased by a factor $G_{a}$, thereby resulting in a relative logarithmic increase in capacity $\left(C_{f}\right)$,

$$
C_{f}=\frac{C^{\prime}}{C} \approx 1+\frac{\log _{2} G_{a}}{\log _{2} \rho}
$$

Resource allocation and utilization: The channel can be visualized to be composed of $\min (\mathrm{M}, \mathrm{N})$ independent and parallel channels (called as "channel modes") in the presence of rich multipath. The data sent on each of these channels is generally referred to as a stream. Each of these channel modes does not have the same gain and hence, whenever a transmitter has a resource for transmission, the strategy is to transmit the signal only on the strongest channel mode (single stream) with all its power ${ }^{5}$.

Scheduling: When a link belongs to more than one otherwise non-overlapping contention regions ${ }^{6}$ (also termed bottleneck link), the total number of transmissions in all those contention regions is limited by the amount of resources at the bottleneck link which is active. On the other hand, if the bottleneck link is passive, then its resources can be overloaded to increase the number of transmissions in each of those contention regions (if possible), thereby increasing the utilization. We refer to this as the passive receiver overloading problem. Hence, careful scheduling of the bottleneck links is essential for increasing the aggregate utilization of the network. Note that, this problem is implicitly addressed in the case of omni-directional and switched beam antennas, since there can be only one transmission in every contention region.

\subsection{MIMO Links}

\subsubsection{Relevant PHY layer characteristics}

Communication pattern: Communication using MIMO links can also be modeled with the notion of "availability of resources". The difference from adaptive arrays is that the available resources (= number of elements, say $K$ ) can not only be used by receivers for suppressing interference, but can also be used by the transmitter to send multiple streams. The objective is still to maximize the SNR of the link. However, the decoding strategy employed at the receiver involves significant complexity.

Potential gains: A MIMO link can provide three types of gain: array gain, diversity gain, and spatial multiplexing gain. If the modulation rates are assumed to be fixed, then array and diversity gains primarily provide range extension as in adaptive array antennas, while spatial multiplexing gain primarily provides higher data rates. While for a transmit array to provide either array or diversity gain, the data streams transmitted from the different antenna elements must be dependent, this is not true for spatial multiplexing. MIMO links provide spatial multiplexing gain in the presence of multipath

\footnotetext{
${ }^{5}$ This requires the knowledge of some CSI at the transmitter.

${ }^{6}$ Every link contends with every other link in the region.
}

or rich scattering, by simultaneously transmitting independent data streams. This gain is defined as the asymptotic increase in the capacity of the link for every $3 \mathrm{~dB}$ increase in SNR [3].

Interference suppression: Similar to adaptive arrays, MIMO links are also capable of flexible interference suppression, with the difference being that the resources saved from suppressing weak and highly correlated interference signals may not only be used to accommodate more transmissions but may directly be used to increase the number of streams being transmitted as well.

\subsubsection{MAC layer considerations}

Leveraging gains: The spatial multiplexing gain can be achieved when the transmit array transmits multiple independent streams of data. When $M=N=K$, the capacity bound of a MIMO link is given by the following equation [2],

$$
C \approx K \log _{2}(1+\rho)
$$

where $\rho$ represents the average SNR at any one receive antenna. On the other hand, when the multiple antennas are used only for array and diversity gain, the asymptotic capacity is

$$
C \approx \log _{2}\left(1+\rho^{\prime}\right)
$$

where $\rho^{\prime}$ is a random SNR with a mean that increases only linearly with the array gain and a variance that decreases with the diversity order. Therefore, capacity grows linearly with $K$ with spatial multiplexing, but only logarithmically with array and diversity gain.

Resource allocation and utilization: Unlike adaptive arrays, MIMO links operate on several streams during spatial multiplexing. Note that the different channel modes in a MIMO link do not experience the same gain. Hence, when a link is allowed to use only a subset of the maximum possible number of streams (say $\mathrm{m}$ out of $\mathrm{K}$ ), it can distribute its transmit power over just the $m$ strongest channel modes (streams). Thus, when compared to two interfering links operating using TDMA at the maximum number of streams $\mathrm{K}$, letting the links operate simultaneously but with the stronger $\frac{K}{2}$ streams will result in improving the overall utilization in the network. We term the gains achievable through such simultaneous operation of interfering but stream controlled links as stream control gains [7].

Scheduling: MIMO links also suffer from the "passive receiver overloading" problem. However, the problem manifests itself in the context of stream control. In a TDMA scheme using all streams $(=D O F s)$ for transmission, if a passive receiver belongs to more than one otherwise non-overlapping contention regions, then there can be an active transmitter in each of those contention regions, overloading the passive receiver with more streams that it can receive. On the other hand in a MIMO MAC employing pure stream control, all the transmitters within a contention region use the best subset of their streams such that no receiver in the region is overloaded. But if any of the receiver nodes also belong to other contention regions, then this prevents the nodes in those other contention regions from transmitting since this will overload the active receiver. This in turn reduces the advantage of spatial reuse resulting in a potential performance degradation and must hence be addressed.

\section{PROBLEM FORMULATION}

A good way of looking at the problem of fair medium access is to first associate each flow with a utility function. The utility function represents the satisfaction measure of the flow corresponding to a parameter of interest. The parameter could be service (throughput), delay, etc. If the utility functions are assumed to be concave, then there exists a mapping between the maximization of the sum of all utility functions and a system-wide notion of fairness. Furthermore, the nature of the utility function chosen determines the model 
of fairness achieved [10]. Hence, the problem of fair channel access equivalently reduces to the optimization problem of maximizing the aggregate utility of the network subject to the transmission constraints with the appropriate choice of the utility function. We adopt the proportional fairness model in this work, wherein a logarithmic (or diminishing returns) utility function is applied to each flow [8]. A good exposition on the motivation for the fairness model can be found in [10]. The transmission constraints are in turn determined by the physical layer properties and the optimization considerations that are specific to the antenna technology.

The focus and contribution of this section is to extend the analytical framework for omni-directional antennas [8] to handle different antenna technologies, and most importantly show how the problem formulation with respect to the different technologies can be accommodated within the same analytical framework. We begin by briefly explaining how the channel access problem in ad-hoc networks can be formulated as a four-phase process generically, and then specifically indicate how the four phases can be adapted with respect to the specific technologies.

\section{Phase 1: Node Graph Generation}

Given the network topology, the node graph $\mathrm{G}=(\mathrm{V}, \mathrm{E})$ is generated as in Figure 1 (a), where V represents the set of nodes in the network, and $\mathrm{E}$ represents the set of edges between all those pairs of nodes that are within transmission/reception range of each other. This does not include edges between nodes that are within carriersense range of each other. The adjacent nodes in Figure 1(a) are assumed to be separated by about $250 \mathrm{~m}^{7}$.

\section{Phase 2: Flow Contention Graph Generation}

From the node graph, the flow contention graph $\mathrm{G}^{\prime}=\left(\mathrm{V}^{\prime}, \mathrm{E}^{\prime}, \mathrm{W}\right)$ is generated as in Figure 1 (b), where V' represents the links in G that have a packet for transmission (active links). E' represents the edges between any two vertices in G' (active links in G) that are within contention range of each other. We define two vertices to be within contention region of each other, if either of their transmissions can cause interference at the other. The $V^{\prime} \times V^{\prime}$ matrix $\mathrm{W}$ represents the weights on the links $[0,1]$ which are representative of the strength of interference between the corresponding active links. We can approximate the weights of the edges in the flow contention graph as ${ }^{8}$,

$$
w_{i j}=\frac{S_{i, j}}{\frac{S_{j}}{S N R_{\text {thresh }}}-N_{j}}
$$

where $S_{i, j}, S_{j}, S N R_{t h r e s h}$ and $N_{j}$ represent the signal strength of the interfering link $i$ on $j$, signal strength of the communicating link $j$, required SNR threshold, and the noise level at the receiver of the desired link $j$. Note that this model can be made more rigorous to include the spatial correlation between the interfering signals in the case of adaptive arrays and MIMO links.

\section{Phase 3: Resource Constraint Graph Generation}

The resource constraint graph G" $=\left(\mathrm{V}^{\prime}, \mathrm{E}\right.$ ") that captures the various contention regions in the network topology based on the flow contention graph, is then generated as in Figure 1 (c). It is essentially a bipartite graph with the two sets of vertices being V' and R, where $V "=V '+R$ and $R$ represents the set of resource vertices, one for each contention region. Each resource vertex could be considered as the resource server for a contention region indicating the total amount of resources $\left(K_{R x}\right.$, without flexible interference sup-

\footnotetext{
${ }^{7}$ The transmission/reception range is assumed to be $250 \mathrm{~m}$ with the carrier sense range to be around $500 \mathrm{~m}$ in the examples that follow in the rest of the paper.

${ }^{8}$ In general, $w_{i j} \neq w_{j i}$ thereby leading to a directed flow contention graph.
}

pression) available in the particular contention region. The links in the set V' have two parameters $\left(K_{T x}, K_{R x}\right)$ indicating the amount of resources available for transmission and reception respectively. The edges in G' correspond to links going from the set V' to set $\mathrm{R}$ indicating the membership of the active links in the various contention regions. These contention regions can be obtained by identifying the various maximal cliques $^{9}$ in the flow contention graph. Figure $1 \mathrm{c}$ ) represents the resource constraint graph for the example considered. It can be seen that there are three maximal cliques $\left\{r_{1}\right.$, $\left.r_{2}, r_{3}\right\}$ that can be identified from Figure $1 \mathrm{~b}$ ), namely $\{\mathrm{abc}$, acd, cdef\}.

\section{Phase 4: Problem Formulation}

Given the resource constraint graph, the channel allocation problem can be modeled as a utility maximization problem subject to the transmission constraints.

For each flow, consider a utility function $U(s)$, for a service (allocation in streams) $s$ that is continuous, differentiable, increasing and strictly concave over the range $s \geq 0$. Also consider some time window $[t-\Delta T, t+\Delta T]$. Let $C_{i, j}(t)$ be the instantaneous channel allocation indicator as a function of time. If $C_{i, j}(t)=m$, then the vertex $i\left(i \epsilon V^{\prime}\right)$ in the flow contention graph has obtained an allocation of $m$ streams in the contention region $j(j \in R)$ at time $t$. Let $a_{i}(t)$ be the channel allocation (service) for flow $i$ in time $[0, t]$.

The model should incorporate the optimization considerations that are specific to the antenna technology. This can be achieved by subjecting the considerations as constraints to the channel access problem. Let $T_{i}(l)$ denote the time for service of $l$ streams for the link $i$. The problem of media access can now be modeled as,

$$
\text { Maximize } \sum_{i} U\left(s_{i}\right), \text { where } s_{i}=\frac{\Delta a_{i}(t)}{\Delta t},
$$

with the allocation being,

$$
\begin{gathered}
\forall t, \forall i, a_{i}(t)=a_{i}(t-1)+C_{i}(t), \\
\text { where } C_{i}(t)=\min \left\{C_{i, j}(t)\right\}
\end{gathered}
$$

subject to the constraints specific to the antenna technology. The above allocation indicates that for a link $i$ to gain channel access in a slot, it must receive non-zero allocation in each of the resource (contention) regions, and the allocation obtained would be the minimal of that obtained over all the contention regions. Since the objective function remains the same, we focus only on the constraint formulation when discussing the last phase with respect to each of the antenna technologies.

\subsection{Omni-directional Antennas}

The node graph is obtained as mentioned before. Figure 1(a) is used as a running example to work through the phases.

The flow contention graph is generated from the node graph. The vertices (links) in V' that are within the reception range of each other have their link weights set to 1 . Links that are out of reception range but inside the carrier-sensing range have a weight between 0 and 1, while those lying outside the carrier-sensing range have their weights set to 0 . Since only one element (resource) is available, as long as two links have an edge of non-zero weight between them, then only one of them can be active in any time slot. Hence, all the edges present in the graph have a weight of 1. Figure 1(b) represents the flow contention graph for the node graph in Figure 1(a) with all the edge weights set to 1 .

The resource constraint graph is obtained such that each of the resource servers has a resource of only one, with the amount of resources available to the links also being limited to one $(K=$

\footnotetext{
${ }^{9} \mathrm{~A}$ clique is a complete subgraph, and a maximal clique is a clique not contained within another clique of larger cardinality.
} 


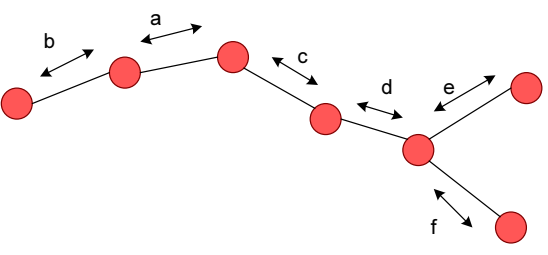

a) Network Topology

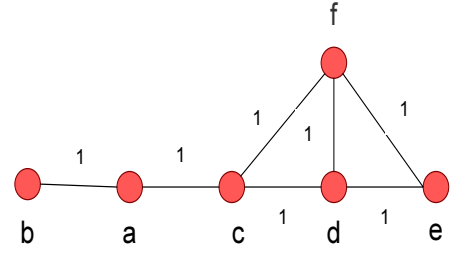

a) Flow Contention Graph

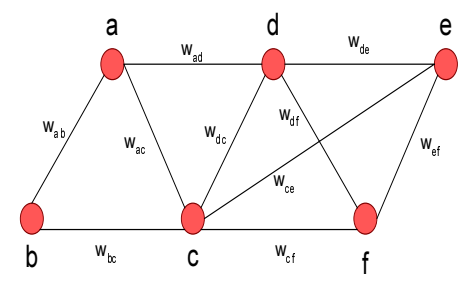

b) Flow Contention Graph

Figure 1: Graphs

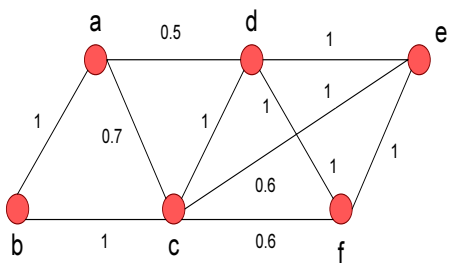

b) Supplement Graph

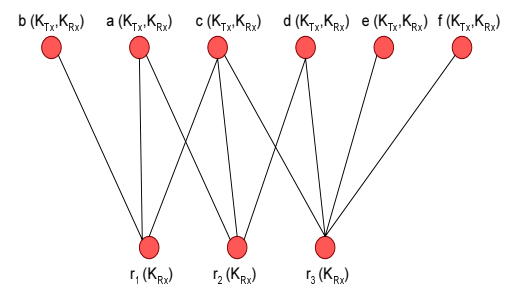

c) Resource Constraint Graph

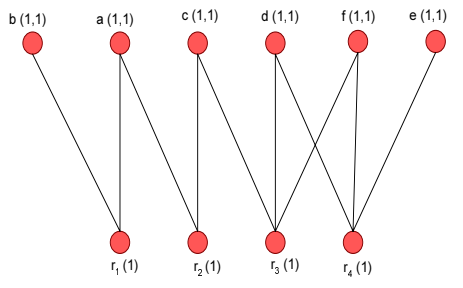

c) Resource Constraint Graph

Figure 2: Switched Beam Graphs

$K_{T x}=K_{R x}=1$ ). Furthermore, there can be only one winner in any contention region since a link can be allotted only an integral number of resources. Figure 1(c) represents the corresponding resource constraint graph for the example considered with $K_{T x}=$ $K_{R x}=1$.

The utility maximization problem is thus subject to the following constraints,

$$
\begin{aligned}
\forall j, & \forall t \sum_{i} C_{i, j}(t) \leq 1, \\
\text { where } C_{i}(t) & =1, \text { if }\left\{C_{i, j}(t)=1\right\}, \forall j \in R \\
& =0, \text { otherwise }
\end{aligned}
$$

The above constraint implies that there can be only one winner in each contention region and a link can gain access to a slot only if it wins in all the contention regions that it belongs to.

\subsection{Switched Beam Antennas}

The node graph is generated as described before in Figure 1(a). However, if range extension is exploited, then the number of edges between nodes in the node graph will accordingly increase.

The flow contention graph is significantly different from Figure 1(b) due to the directionality involved. It is presented in Figure 2(a). For a given traffic pattern, the active links in the network are determined. Now, two links are said to have an edge between them, if either of the receivers is within the reception/sensing range of the transmitters of both the links on the same beam, in the case of uni-directional communication. In the case of bi-directional communication, both the ends of a link must be considered as a receiver in deciding if it contended with another link. Further, if the directional gain is to be used for range extension, then the corresponding range extension factor can be obtained from equation 3 and the flow contention graph can be generated taking into account the extended range of communication and interference in the node graph. The weights on the edges between links are still $\{0,1\}$ due to the lack of fine grained interference suppression. The incorporation of directionality in the generation of the flow contention graph, exploits the additional degree of spatial reuse resulting from directional transmissions by reducing the number of edges between links. However, the flow contention graph does not account for the effect of side lobes, to model which we use another flow contention graph which we refer to as the supplement graph hereafter. The supplement graph is essentially the omni-directional flow contention graph for the same network graph (see Figure 2(b)).

The resource constraint graph is generated in the same manner as in the omni-directional case. However, to model the effect of side lobes, we consider every node to have a main beam with a peak gain of unity and side lobes of a smaller but uniform gain along the other $K-1$ beam directions (a conical main lobe and a spherical side lobe) with a front-side lobe ratio, $f(f>1)$. . Hence, whenever a link is scheduled its neighbors that lie along the beam direction in the supplement graph are updated with an increased noise power in a diametrically opposite beam. Whenever a link is to be scheduled in the flow contention graph its total noise power along all the beam directions is accumulated from the supplement graph to check if the SNR threshold is still maintained (see equation 18). If so, then the link is scheduled. The constraints to the problem can now be formulated as,

$$
\begin{aligned}
& \forall j, \forall t \sum_{i} C_{i, j}(t) \leq 1, \\
\text { where } C_{i}(t)= & 1, \text { if }\left\{C_{i, j}(t)=1\right\}, \forall j \epsilon R \\
& \& \& \sum_{m=0}^{K-1} \frac{N_{i}(m) \cdot w_{i j}}{f} \leq \frac{1}{S N R_{\text {thresh }}} \\
= & 0, \text { otherwise }
\end{aligned}
$$

where $K$ is the total number of elements (beams), $w_{i j}$ is the interference weight between the links $i$ and $j$, and $N_{i}(m)$ is a variable corresponding to link $i$ such that,

$$
N_{i}(m)=l \text {, if } \exists l \text { transmissions along beam direction } m
$$

If instead of range extension, a direct increase in capacity is required by switching to higher modulation rates, then the resource in each contention region can be viewed to be scaled by a factor $\left(C_{f}\right.$, given in equation 5) with each link's allocation being limited to $\left\{0, C_{f}\right\}$.

\subsection{Fully Adaptive Array Antennas}

The node graph is the same as in the other antenna technologies (Figure 1(a)). But if range extension is employed, the number of edges would increase. 

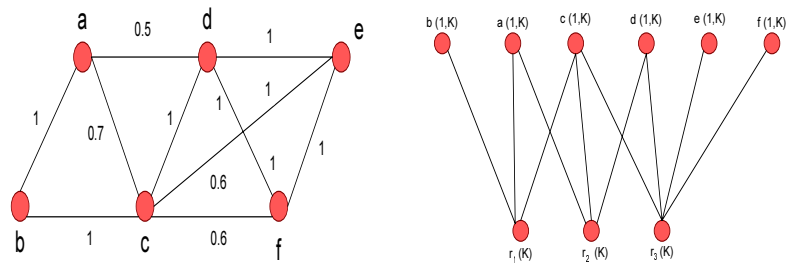

a) Flow Contention Graph b) Resource Constraint Graph

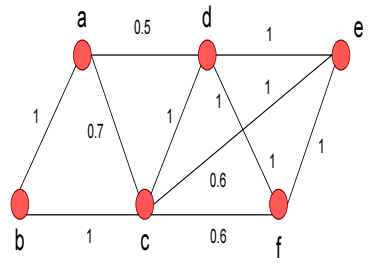

a) Flow Contention Graph

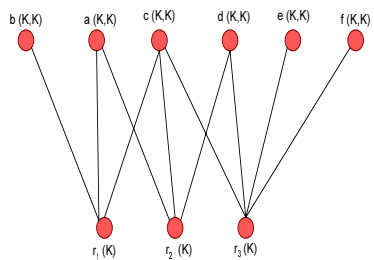

b) Resource Constraint Graph

Figure 3: Adaptive Array Graphs

The flow contention graph is generated in the same way as for the omni-directional antennas. However, since the adaptive array antennas are capable of fine grained interference suppression, the edges between the links can have weights between 0 and $1(0 \leq$ $w_{i j} \leq 1$, see Figure 3(a)). Also, the range extension resulting from the array gain (see equations 6 and 7) can be incorporated in the generation of the flow contention graph.

The procedure for the resource constraint graph generation remains the same. However, the resource servers have a total of $K$ (= number of elements) resources each, with only one resource being used by any link for its own transmission. The remaining resources are used by the link for flexible interference suppression $\left(K_{T x}=1, K_{R x}=K\right)$. The resource constraint graph is presented in Figure 3(b).

The constraints for adaptive array antennas are thus,

$$
\begin{gathered}
\forall j, \forall t \sum_{i} C_{i, j}(t) \leq 1, \\
\text { where } C_{i}(t)=1, \text { if }\left\{C_{i, j}(t)=1\right\}, \forall j \epsilon R \\
=0, \text { otherwise } \\
G\left(s_{1}\right) \geq G\left(s_{2}\right) \geq \cdots \geq G\left(s_{K}\right) \\
\forall t, \forall i \sum_{l} w_{l i} C_{l}(t) \leq K, \text { if } C_{i}(t) \geq 0, \\
\text { where } l \epsilon L, \text { the set of links such that } \\
\exists \operatorname{some} j \epsilon R, \text { for which }\left\{(l, j) \epsilon E^{\prime \prime} \& \&(i, j) \epsilon E^{\prime}\right\} \\
\max \left(T_{i}(l)\right)<\max \left(T_{j}(l)\right), \text { for a given service } l, \\
\text { where } i \epsilon \text { RE } D, j \epsilon \text { W HITE }
\end{gathered}
$$

If the streams are arranged in the descending order of their gains $G\left(s_{i}\right)$, then the constraint in equation 20 ensures that the strongest stream will always be chosen. Constraint in equation 21 incorporates the flexible interference suppression possible through adaptive array antennas. This in turn, creates more resources and hence more transmissions in the same contention region if the interference caused by some links is weak. Thus, while the total number of resources used in any contention region can potentially be greater than $K$, the total number of resources used up by any link in any contention region cannot exceed $K$. If the array gain is to be used towards increasing the capacity directly rather than increasing the range, then the resource in each contention region $(K)$ can be viewed to be scaled by a factor $\left(C_{f}\right.$, given in equation 9$)$ with each link's allocation being limited to $\left\{0, C_{f}\right\}$.

To enable passive receiver overloading it becomes necessary to identify links belonging to multiple contention regions ("red links") from those that belong to a single contention region ("white links"). The procedure adopted to color the links is explained in the following section. The last constraint ensures that a two level scheduling of the red and white links is performed, with the red links being scheduled before the white links. This would help the white links maximize the utilization when scheduled separately by avoiding the problem of passive receiver overloading, and also help achieve proportional fairness.
Figure 4: MIMO Links Graphs

\subsection{MIMO Links}

The node graph generation remains the same as in the other cases (Figure 1(a)).

The flow contention graph is generated in the same manner as in the case of adaptive array antennas (Figure 4(a)).

The resource constraint graph is similar to the one in adaptive array antennas, except that every link can potentially use all its $K$ resources for transmission as shown in Figure 4(b) $\left(K_{T x}=K_{R x}=\right.$ $K)$. Coloring is once again required to enable passive receiver overloading. Only the white links perform stream control, while the red links operate on all available $K$ streams.

The problem of media access now has the following constraints,

$$
\begin{gathered}
\forall j, \forall t \sum_{i} C_{i, j}(t) \leq K, \\
\text { where } C_{i}(t)=\min \left\{C_{i, j}(t)\right\} \\
0 \leq C_{i, j}(t) \leq K \text { if } i \epsilon W H I T E, \text { and } \\
C_{i, j}(t)=\{0, K\} \text { if } i \epsilon R E D \\
G\left(s_{1}\right) \geq G\left(s_{2}\right) \geq \cdots \geq G\left(s_{K}\right) \\
\forall t, \forall i \sum_{l} w_{l i} C_{l}(t) \leq K, \text { if } C_{i}(t) \geq 0, \\
\text { where } l \epsilon L, \text { the set of links such that } \\
\exists \operatorname{some} j \epsilon R, \text { for which }\left\{(l, j) \epsilon E " \& \&(i, j) \epsilon E^{\prime}\right\} \\
\max \left(T_{i}(l)\right)<\max \left(T_{j}(l)\right), \text { for a given service } l, \\
\text { where } i \epsilon R E D, j \epsilon \text { W HITE }
\end{gathered}
$$

The primary concern of the model is to perform the two-level colored scheduling to eliminate the under-utilization that results due to the bottleneck links. This is captured in the last set of constraints where the red links are scheduled before the white links, and when scheduled they receive all $K$ streams as allocation. On the other hand the white links that get scheduled later can receive service anywhere between 0 and $K$ streams depending on the fair share in their maximal clique, due to stream control. If the streams are arranged in the descending order of their gains $G\left(s_{i}\right)$, then the constraint in equation 30 ensures that the best streams are chosen by the white links. Note that the amount of resources used up at the resource server is determined by the edge weights in the flow contention graph and hence the gain of flexible interference suppression is automatically leveraged by way of more resources being available at the resource servers due to weak interference.

\section{UNIFIED MAC FRAMEWORK}

The objective of the centralized algorithm is to maximize the aggregate utilization of the network subject to the transmission constraints and conforming to the fairness model. Coming up with a feasible ${ }^{10}$ schedule that also maximizes utilization is a well-understood problem. The pseudo-code for the outline of the proposed solution

\footnotetext{
${ }^{10}$ Satisfying the constraints and conforming to the fairness model.
} 
is presented in Figure 5 and consists of three components: (i) The first component generates the flow contention graph (Step 1) and determines all the contention regions (maximal cliques) in the flow contention graph and arrives at the minimum clique cover. Maximal cliques define distinct contention regions in the network where there can be only as many winners in any contention region as to not exceed the number of resources in the region. (ii) The second component colors the links. The fairness model that we employ is proportional fairness. A property of proportional fairness in shared wireless medium access is that the solution tends to favor links that belong to a single contention region (non-bottleneck links) from those that belong to multiple contention regions (bottleneck links). Hence, it becomes vital for the algorithm to identify the bottleneck links from the non-bottleneck links and schedule them appropriately to ensure proportional fairness. We refer to this process as coloring (Step 2). (iii) Once the maximal cliques are obtained and the vertices of the flow contention graph are colored, the final component of the algorithm schedules the links within and across the slots, satisfying the constraints and adhering to the fairness model (Step 3).

To begin with, we explain these three components generically and then discuss the details with respect to the specific antenna technologies.

\subsection{Clique identification}

Identifying all the maximal cliques in a graph is known to be an NP-Hard problem. Hence the centralized algorithm makes use of an algorithm that is capable of determining all the maximal cliques in chordal graphs (having cycles of size $<4$ ) in linear (in the number of vertices) time. It first determines the perfect elimination ordering (PEO) using LexBFS (Lexicographic Breadth First Search) [11] for the chordal graph and then applies a linear algorithm that detects all the maximal cliques given the PEO using a theorem by Fulkerson and Gross [12].

Though the algorithm works only for graphs having cycles of size less than four, note that the graph in our case corresponds to the flow contention graph. Hence for a cycle of size four to be present in the flow contention graph, a cycle of atleast size eight must be present in the node graph with no nodes being present inside the cycle, which is an unlikely scenario. For the flow contention graph in Figure 1(b), the different maximal cliques are $c d e f, a b c$ and $a c d$.

\subsection{Coloring}

To be able to color the links, it is first necessary to rank them. Every vertex (link) has two attributes $(d, s)$ : clique degree $d$ (number of maximal cliques that the vertex belongs to) and maximum size $s$ of all possible cliques that it belongs to. The vertices are ranked lexicographically based on the tuple $(d, s)$ with the vertex having the highest degree ranked first, and the maximum size $s$ is used to break ties. However, it is not necessary to rank vertices that have a degree of one.

Initially all the vertices are colored white ${ }^{11}$ (line 1, see Figure 5). Based on the tuple information $(\mathrm{d}, \mathrm{s})$ for each vertex, the vertices are ranked lexicographically as described before (lines 2-3). Then the vertex with the highest rank is recursively chosen, and colored red, following which, the particular red colored vertex and edges emanating from it are removed from G' (lines 4-5). The tuple (d,s) of the remaining vertices in $G^{\prime}$ are updated and the remaining vertices are re-ranked once again (line 6). The process repeats until no more vertices can be colored red (line 7).

In Figure 1(b), vertex $c$ obtains the highest rank with a degree of 3 , followed by $d$ that as a degree of 2 and a maximum size of 4 .

\footnotetext{
${ }^{11}$ A white (red) vertex in G' corresponds to a white (red) link in the network.
}

Vertex $c$ is colored red first, followed by vertex $d$ in the next cycle. The rest of the vertices remain colored white.

\subsection{Schedule}

The number of resources that can be used by any link for its transmission $\left(K_{T x}\right)$ and the total number of resources that can be tolerated in any contention region $\left(K_{R x}\right)$, vary from one antenna technology to another. If slot is considered to be the basic time unit, then we broadly divide the time axis into units that we refer to as slot sequences (slot_seq). A slot sequence consists of the minimum number of slots required to provide a service of atleast $K_{T x}$ to every link in the network. We identify the slot sequence with respect to the different antenna technologies subsequently (line 9 in Figure 5). The schedule repeats after every slot sequence. Within every slot sequence, we execute a two-level scheduling of the red and the white links. The red links are scheduled first followed by the white links and the switching between the red and white links is done in accordance such that at the end of the slot sequence every link has obtained a service of atleast $K_{T x}$. The two-level scheduling along with the appropriate switching conditions ensure that the resulting scheduling vector in a slot sequence is proportionally fair (formal proof in [13]). Hence, as long as time is considered as an integral multiple of slot sequences, the resulting scheduling vector will also be a proportionally fair one.

Within a slot sequence, the schedule begins with the red links (line 11). The red links always transmit on only $K_{T x}$ resources. Hence, the subset of red links not having received an allocation of $K_{T x}$ resources is chosen subject to the conditions that they have atleast $K_{T x}$ resources with them and their neighbors have enough resources to suppress their transmission. Then the red link with the minimum service obtained thus far is scheduled from the subset (line 14) and the resources at the concerned link and the neighboring links are updated to reflect this transmission (lines 33-34). The strength of the interference between the pairs of links is taken into account in the update of resources. As many red links from the subset chosen are scheduled in this fashion, as long as the link and its neighbors have sufficient resources. When no more red links can be scheduled in the same slot, in order to maximize the utilization, white links are attempted to be scheduled. The white links can transmit with any number of resources in the range $\left[1, K_{T x}\right]$. Hence, they are allotted one resource after another. This granularity of allocation for the white links makes it possible for several white links in the same contention region to transmit simultaneously (provided $K_{R x}>1$ ). As in the case of red links, the subset of white links, each with sufficient resources both at its neighbors as well as itself for a single stream transmission, is chosen (line 16). The white link with the minimum service is then scheduled and the resources are updated at the link and its neighbors (lines 24 and 35-37). The process continues until all possible white links are scheduled with as many resources as possible. The schedule then moves to the next slot after re-setting the parameters (line 30).

When the red link with minimum service exceeds the white link with minimum service, the schedule switches to the white links (line 21). The white links are now scheduled as before (lines 22-25), and when all possible white links have been scheduled, the red links are attempted to be scheduled (lines 26-29). The subtle difference from before is that when the red links (chosen from the subset of red links with available resources) are scheduled in the same slot as the white links, the red link with maximum rank number (lowest in rank) is scheduled instead of the red link with the lowest service (lines 3234). By serving the red link with the lowest rank, we eliminate lesser contention regions and hence try to maximize the utilization in the slot. The procedure continues until the white link with minimum service has exceeded the red link with maximum service. 


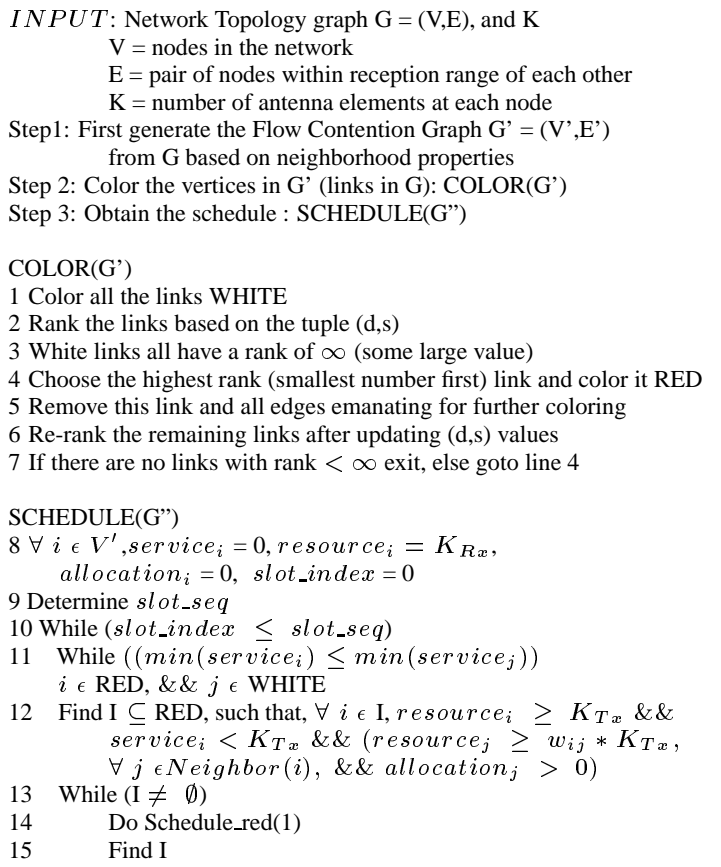

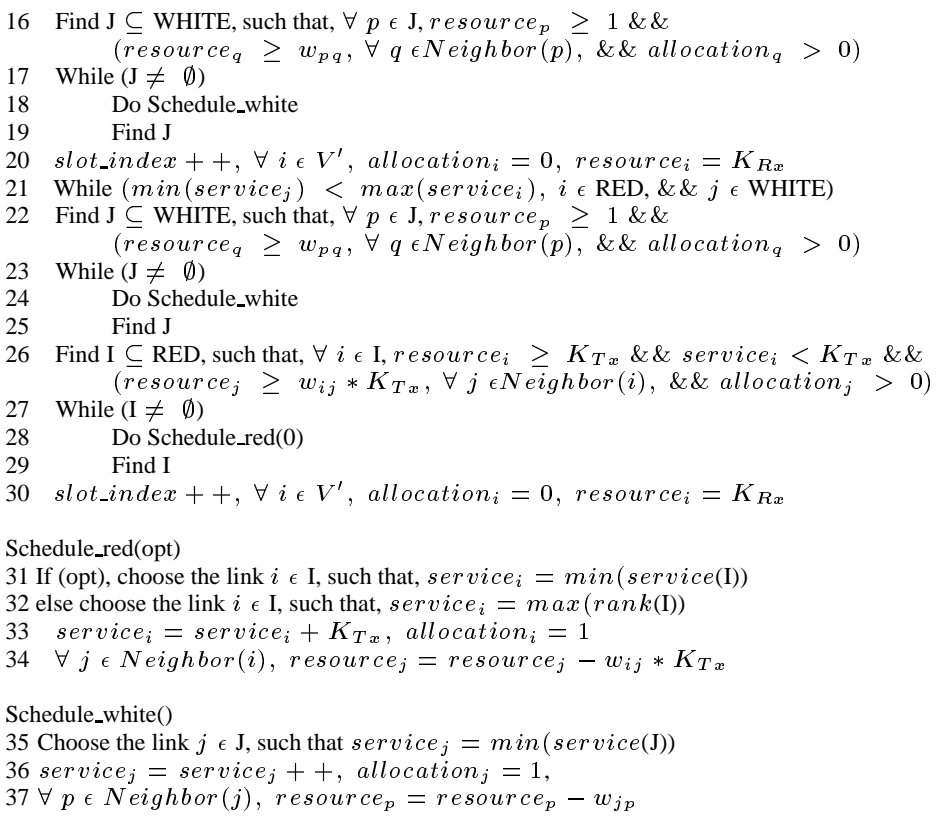

\section{Figure 5: Pseudo Code for Centralized Algorithm}

In the rest of this section, we outline how the resources are utilized in each contention region with respect to each of the antenna technologies and also show how the resource constraints are met and the optimization considerations identified in Section 3 are leveraged.

\subsection{Instantiations}

\subsubsection{Omni-directional Antennas}

Every link can transmit and receive on only one resource $\left(K_{T x}=\right.$ $1)$ and every contention region also has only one resource $\left(K_{R x}=\right.$ $1)$. The slot sequence is thus determined by the largest maximal clique in the flow contention graph since the transmission by one link prevents all the other links in the same contention region from transmitting in the same slot. The weights of all the edges in the flow contention graph are one, and hence transmission by any link will reduce the resources of all the other links in the same contention region to zero. For this reason, it is not essential to distinguish between the red and white links. Hence, all the links can be colored red without going through the elaborate process of finding all the maximal cliques and only the part of the algorithm in Figure 5 that is relevant to the red links will be triggered (lines 8-15 and 31-34). In scheduling a red link within a slot, the one with the minimum service is chosen, which is achieved by setting the parameter opt to 1 (line 14).

\subsubsection{Switched-beam Antennas}

The flow contention graph is generated for the given traffic pattern taking directionality into account. For a $K$ element array, $K$ beams of $\frac{360}{K}$ degrees each are considered. However, the presence of scattering limits the gains obtained from increasing $K$, and must be accounted for in the generation of the flow contention graph. Every link and contention region in a switched-beam environment also has only one resource $\left(K_{T x}=K_{R x}=1\right)$. Thus the slot sequence is again given by the size of the largest maximal clique in the flow contention graph. As before, all the links in switched-beam can also be colored red and hence only the aspects of the algorithm relevant to the red link scheduling are invoked (lines 8-15 and 31-34 with opt set to 1). Since switched-beam links do not perform flexible interference suppression, the weights of the edges in the flow contention graph are all set to one. Hence, the transmission by one link reduces the resource at each of the neighboring links in the flow contention graph to zero (line 34 with $w_{i j}=1$ and $K_{T x}=1$ ). The side lobes are accounted for in the supplement graph. Each link $j$ in the supplement graph also maintains a resource variable resource $_{j, \text { sup }}$ which is initially set to $\frac{1}{S N R}$. Whenever a link $i$ is scheduled, the resource of all the neighboring links $(N(i))$ in the supplement graph is diminished by the appropriate noise power $\left(\frac{w_{i j}}{f}, f\right.$ being the frontside lobe ratio) as in,

$$
\text { resource }_{j, \text { sup }}=\text { resource }_{j, \text { sup }}-\frac{w_{i j}}{f}, \forall j \in N(i)
$$

Thus, whenever a link $i$ is a candidate to be scheduled in the flow contention graph due to the availability of resource (resource $_{i}=$ 1 ), then its corresponding resource $\left(\right.$ resource $_{i, \text { sup }}$ ) in the supplement graph is also checked. If it happens to be greater than zero then the link is scheduled.

If range extension is desired, then this is incorporated in the generation of flow contention graph that is fed to the algorithm. On the other hand, if capacity increase is desired, then the resource of each link and contention region $\left(K_{T x}=K_{R x}=1\right)$ can be viewed to be increased by the factoral increase in capacity $\left(C_{f}\right.$, given by equation 5 ). But the resource in the supplement graph remains unchanged and is totally decoupled from the resource in the flow contention graph. 


\subsubsection{Adaptive Array Antennas}

The flow contention graph that is fed as input to the algorithm is the same as in the omni-directional case. However, the number of resources handled by each link changes. Every link is capable of transmitting on only one resource as in the previous cases, but can handle (suppress) upto $K-1$ resources ( $K$ being the number of antenna elements). Though the transmission resource per link $\left(K_{T x}\right)$ is one, the resources per contention region $\left(K_{R x}\right)$ is $K$. Since several links in a contention region can transmit in the same slot based on the availability of resources, the slot sequence will be in the range of $\left[\frac{\text { max_clique }}{K}\right.$, max_clique $]$ and can be determined from the flow contention graph. The possibility of multiple winners in a contention region calls for coloring and two level scheduling to ensure a proportionally fair vector as well as to enable passive receiver overloading. Hence, all components of the algorithm presented in Figure 5 are relevant to adaptive arrays, with the opt parameter being set to 0 in line 28 based on the reasoning provided in the earlier part of the section. The flexible interference suppression characteristic of adaptive arrays is taken care of in the update of resources (lines 34 and 37) where the appropriate edge weights $\left(w_{i j}\right)$ are used in deciding the amount of resources required to be sacrificed for suppressing interference. Further, since the algorithm tries to use up all the resources in a contention region by appropriately scheduling transmissions, the gains from diversity are not going to be significant. However, the array gain can be still be used for range extension or capacity increase similar to the directive gain in switched beam antennas. In the case of capacity increase, the resource at each link and contention region $(1, K)$ can be viewed to increase by the factoral increase in capacity $\left(C_{f}\right.$, as in equation 9$)$.

\subsubsection{MIMO Links}

The critical difference in MIMO links from that of adaptive arrays is that the number of resources that can be transmitted by any link $\left(K_{T x}\right)$ can also be $K$, in addition to the amount of resources in a contention region $\left(K_{R x}\right)$ being $K$. In order to be able to leverage the advantages of stream control, without suffering from the disadvantages of passive receiver overloading problem, coloring of the links is performed (lines 1-7). The red links are always made to transmit on all $K$ resources (line 33) while its only the white links that operate on $[1, K]$ resources. Hence, the white links alone perform stream control by sharing the resources in the contention region and operating simultaneously. Thus the slot sequence required for all the links to achieve an allocation of atleast $K_{T x}$ resources reduces to that of the largest maximal clique as in the case of omni-directional and switched-beam antennas. The resources in a contention region are allotted to the white links in the contention region one by one (line 36) until all the resources are used up, so that the gains of stream control are maximized. Further, since the white links in a contention region share all the resources in the region (lines 35-37), scheduling of red links during the white link scheduling (lines 2629 and 31-34 with $o p t=0$ ) does not arise. Also, the update of resources is performed based on the actual weights of the edges in the flow contention graph, and hence leverages the advantages of flexible interference suppression as in adaptive arrays (lines 34 and 37).

\section{PERFORMANCE INSIGHTS}

In this section, we evaluate the performance of the different antenna technologies under different network configurations and derive insights from the study. We present results for the technologies evaluated over a network configuration of 100 nodes in $1500 \mathrm{~m} *$ $1500 \mathrm{~m}$ area. The results for other network configurations are omitted due to lack of space. We consider the following components: (i) impact of scattering, (ii) impact of number of elements, and (iii) impact of load. Each of these components are viewed from the perspectives of, when the available antenna gains are used towards range extension, and when they are used for capacity (rate) increase.

We use an event-driven packet level simulator. We consider different angles of scattering from 45 degrees to as high as 180 degrees to study the impact of different degrees of multipath in the network. The number of elements used is from the set $\{1,4,6,8,12\}$. The load on the network can be anywhere from 5 flows to 40 flows from the set $\{5,10,20,30,40\}$. We primarily use throughput (transmissions/slot) as the metric of evaluation. We use the antenna pattern model used in [5] for the main lobe, and side lobe gains of switched beam antennas. Further, the SNR on each of the link is assumed to be $10 \mathrm{db}$ in order to achieve a specific level of probability of error on the link. Each of the simulations is run for 500 time slots. Every data point in the graphs presented subsequently is averaged over 10 random seeds. Mobility is not considered in our simulations. The flows in the network are all multi-hop flows and are considered to be back-logged throughout the simulation. Each of the flows is generated with a random source-destination pair. The routes for the flows are obtained using a shortest path routing protocol and fed as input to the algorithm to perform scheduling for channel access.

\subsection{Performance Trade-offs}

\subsubsection{Impact of Scattering}

We first consider the performance of switched beam antennas in the case of LOS. In this case, MIMO links should change their strategy to operate as an adaptive array since spatial multiplexing cannot be leveraged in LOS. Figure 6 shows the performance of switchedbeam and adaptive arrays in LOS environments for varying number of elements and flows respectively for both the cases of range extension and rate increase. The gain in adaptive arrays over the switched beam antennas is due to its flexible interference suppression capabilities and also due to the degradation suffered by switched beam antennas from scalloping loss. The degradation due to scalloping loss would be even more significant in mobile environments.

Observation 1: In the presence of LOS, adaptive beamforming provides the best performance. MIMO links cannot leverage spatial multiplexing; hence change of strategy is required for MIMO.

Figure 7 presents the performance of switched beam antennas in varying degrees of multipath. Adaptive arrays are considered for reference in these results. In the presence of multipath, adaptive arrays are not significantly impacted as long as each link is able to control the number of transmissions in its neighborhood. However, switched beam antennas suffer significantly with increasing multipath. In the presence of multipath, beyond a certain number of elements (determined by the scattering angle) the performance tends to saturate, and the knee of the saturation point keeps shifting to the left with increasing multipath.

Observation 2: Switched beam antennas suffer from multipath unlike adaptive arrays and MIMO links.

\subsubsection{Impact of Number of Elements}

The environment considered is a multipath environment with a scattering angle of 60 degrees. The load in the network is maintained a constant of 20 flows while the number of elements is varied. Each of the flows is a multi-hop flow with an average of 4-5 hops each. The results are presented in Figures 8(a) and (c). The following observations can be made: (i) The MIMO links outperform all the technologies under consideration, owing to the potential linear increase in capacity and the optimization considerations. The im- 


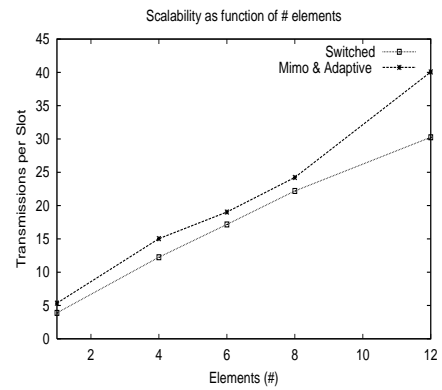

a) Varying Elements: Range Extension

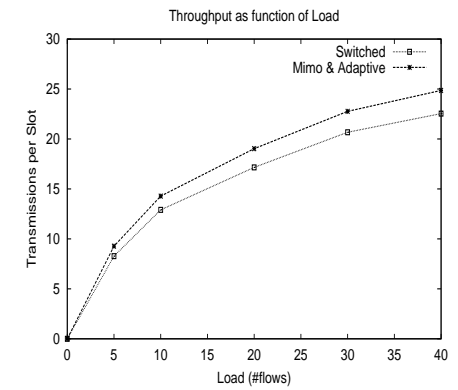

b) Varying Load: Range Extension

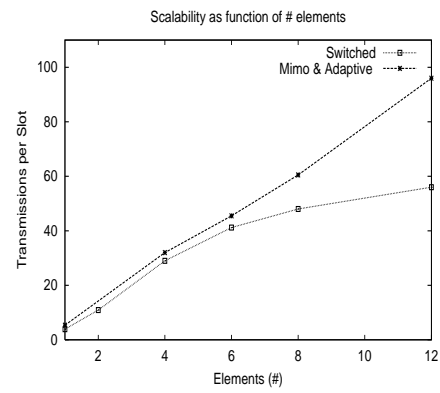

c) Varying Elements: Rate Increase

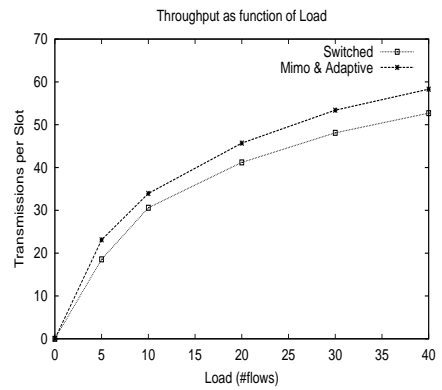

d) Varying Load: Rate Increase

Figure 6: Line of Sight

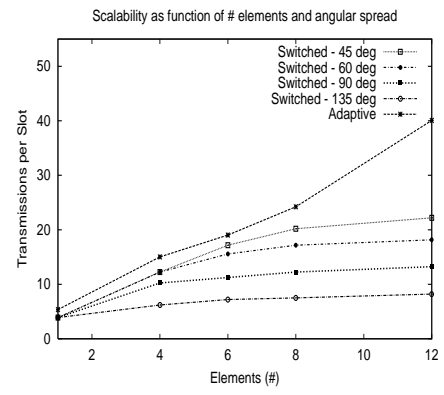

a) Varying Elements: Range Extension

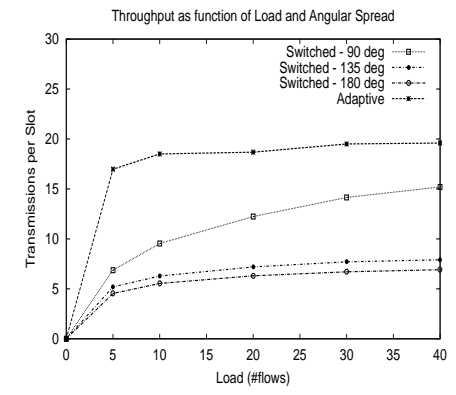

b) Varying Load: Range Extension

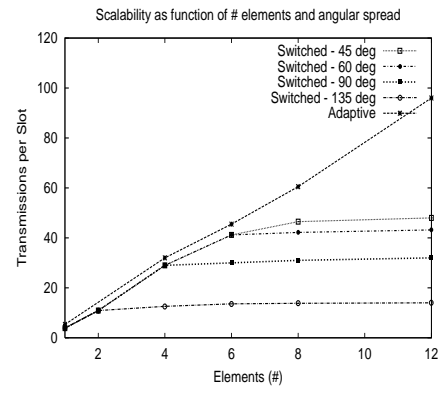

c) Varying Elements: Rate Increase

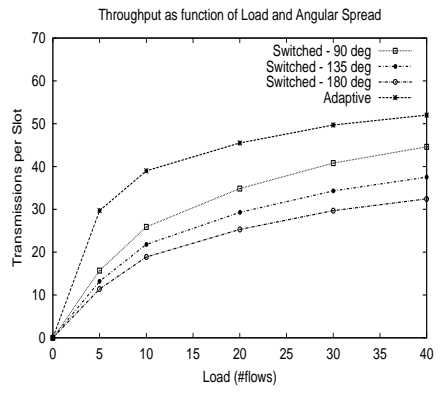

d) Varying Load: Rate Increase

Figure 7: Impact of Scattering

provement over the adaptive array antennas is due to the fact that MIMO links employ spatial multiplexing to exploit all available resources efficiently. A potential reason for the inefficient resource utilization in adaptive array antennas stems from cases where the number of links in a contention region is less than the number of elements. (ii) Switched beam's performance tends to saturate beyond the use of six elements due to the scattering angle of 60 degrees.

When range extension is exploited, with an increase in the number of elements, the number of active neighboring links to any link considered also increases due to the increased range. Hence, it is possible for the per-source throughput to scale (with number of nodes) as long as the number of elements is sufficient to hit full network connectivity and accommodate all transmissions in the network. While this is potentially true for adaptive arrays and MIMO links, the presence of strong multipath could saturate switched-beam's throughput without helping it to scale. In the case where rate increase is leveraged, the number of active neighboring links does not change. But employing higher rates increases the link capacity logarithmically (with elements) in the case of switched beam and adaptive, and linearly in the case of MIMO links. Again, in the case of switched beam antennas, strong multipath would limit its performance.

Observation 3: Average number of active neighboring links is an influential parameter in performance. In range extension, increasing elements increases the number of active neighboring links, unlike in rate increase where the gain comes purely from employing higher rates.

\subsubsection{Impact of Load}

The number of elements is fixed at six and the load in the network is increased upto 40 multi-hop flows. The scattering angles are considered only from 90 degrees onwards, since the impact of scattering on a six element link is felt only from 90 degrees onwards. The re- sults are shown in Figures 8(b) and (d). The MIMO links exhibit the best performance. Since the number of elements is fixed, the transmission ranges are fixed. However, increasing the load increases the number of active neighboring links to any link considered. In the case of adaptive arrays and MIMO links, when the number of active neighbor links increases beyond $K$ (= number of elements), the throughput starts to saturate (transmissions in the contention region bounded by $K$ ). Further, when the load is light and the number of active links in a contention region is less than $K$, then the throughput in the contention region and hence performance is bounded by the number of active neighboring links for adaptive arrays, while it is still bounded by $K$ in the case of MIMO links. In the case of switched beam antennas, more than the number of active neighboring links, it is the orientation of communication of the neighboring links that matter. Hence, more than $K$ transmissions can possibly be scheduled in the same region thereby showing a slight increasing trend in performance with increasing load. But for a given $K$, the transmissions in a contention region can be viewed to be bounded by the number of active neighboring links. However, the reduction in performance due to multipath is much more significant. Also, they are not capable of performing flexible interference suppression unlike adaptive arrays and MIMO links.

Observation 4: Increasing the load increases the number of active neighboring links; but performance is bounded by the number of links that can be accommodated in a contention region, which in turn is determined by the number of elements.

\subsubsection{Range Extension vs. Rate Increase}

From the results presented thus far in Figures 6, 7 and 8, it can be clearly seen, that the performance improvements obtained from using the gains towards rate increase are far more than those obtained from using the gains for range extension. This is possibly because, when the gains are used for range extension, with a decrease in the 
Scalability with \# elements

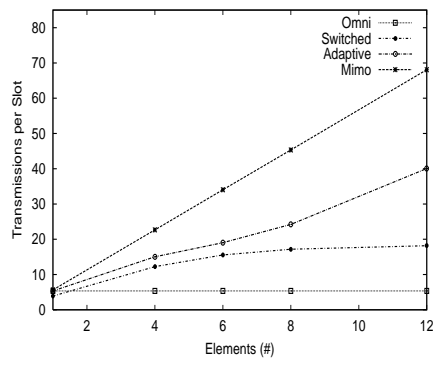

a) Varying Elements: Range Extension

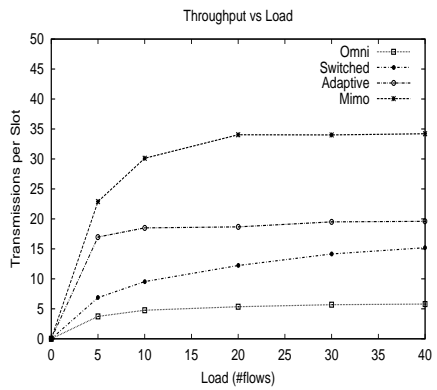

b) Varying Load: Range Extension

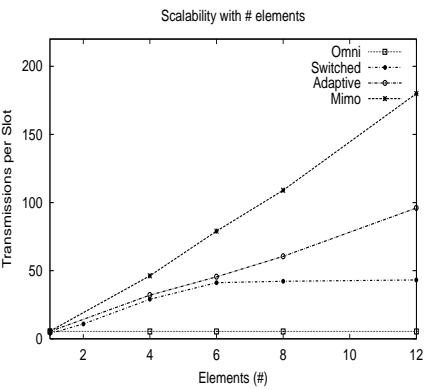

c) Varying Elements: Rate Increase

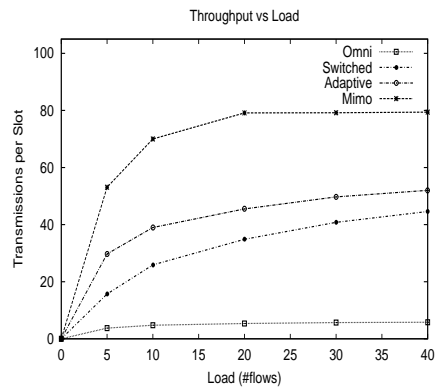

d) Varying Load: Rate Increase

Figure 8: General Performance Trade-offs

number of hops resulting from increased range, the spatial reuse also decreases. However, when the gains are used for rate increase, the spatial reuse is retained while the capacity is increased. However, the observation that rate increase always provides better gains than range extension, may not be true for all the antenna technologies. In fact, in [13] we show that there exist conditions in the case of adaptive arrays and switched beam antennas for this to hold true.

\section{RELATED WORK}

To the best of our knowledge, we are not aware of any related work in literature, that performs a systematic study of the different antenna technologies in the context of medium access control in ad hoc networks. While there have been in-numerous works at the PHY layer with respect to the antenna technologies, work at the MAC layer is restricted predominantly to developing distributed protocols for switched beam antennas. To name a few [4-6] aim to address the issue of distributed medium access control in ad-hoc networks with switched beam antennas, while [14] considers cellular scenarios in which the base-stations are equipped with smart antennas. The goal in [5] was to estimate a lower bound on the overall performance of an ad-hoc networks with switched beam antennas. [4] and [15] are works that exploit the directive gain provided by directional antennas for purposes of range extension and minimization of power consumption respectively.

\section{CONCLUDING REMARKS}

In this work, we have attempted to bring together the modeling of medium access control for ad hoc networks with different antenna technologies under a common framework and use it towards the design of unified centralized algorithms. The distributed counterparts are presented in [13] due to lack of space here. Finally, the algorithms are used to provide insights into the performance of the different technologies. There are some issues existing as part of our future work: (a) OL-MIMO with antenna selection is an alternative to CL-MIMO with stream control, that gives lesser improvement but significantly reduces overheads; (b) Modeling of range extension resulting from the simultaneous use of multiple streams;(c) Optimization considerations in the presence of mobility, where the significance of diversity gains is increased; and (d) In an orthogonal direction, using the antenna gains toward power optimization.

\section{REFERENCES}

[1] J. H. Winters and M. J. Gans, "The Range Increase of Adaptive Versus Phased Arrays in Mobile Radio Systems," IEEE Transactions on Vehicular Technology, vol. 48, no. 2, pp. 353-362, Mar 1999.
[2] D. Gesbert, M. Shafi, D. Shiu, P. J. Smith, and A. Naguib, "From Theory to Practice: An Overview of MIMO Space-Time Coded Wireless Systems," IEEE JSAC, vol. 21, no. 3, pp. 281-301, Apr 2003.

[3] G. J. Foschini and M. J. Gans, "On Limits of Wireless Communications in a Fading Environment When Using Multiple Antennas," Wireless Personal Communications, vol. 6, pp. 311-335, 1998.

[4] R. R. Choudhury, X. Yang, R. Ramanathan, and N. H. Vaidya, "Using Directional Antennas for Medium Access Control in Ad Hoc Networks," in Proc. of ACM MOBICOM, Sept. 2002.

[5] R. Ramanathan, "On the Performance of Ad Hoc Networks with Beamforming Antennas," in Proc. of ACM MOBIHOC, Oct. 2001.

[6] T. Korakis, G. Jakllari, and L. Tassiulas, "A MAC Protocol for Full Exploitation of Directional Antennas in Ad-hoc Wireless Networks," in Proc. of ACM MOBIHOC, June 2003.

[7] M. F. Demirkol and M. A. Ingram, "Stream Control in Networks with Interfering MIMO Links," Proc. of IEEE WCNC, Mar. 2003.

[8] T. Nandagopal, T-E. Kim, X. Gao, and V. Bhargavan, "Achieving MAC Layer Fairness in Wireless Packet Networks," Proceedings of ACM MOBICOM, Aug 2000.

[9] J. B. Anderson, "Array Gain and Capacity for Known Random Chnanels with Multiple Element Arrays at Both Ends," IEEE Journal on Selected Areas in Communications, vol. 18, no. 11, pp. 2172-2178, Nov 2000.

[10] F. P. Kelly, A. Maulloo, and D. Tan, "Rate Control in Communication Networks: Shadow Prices, Proportional Fairness and Stability," Journal of the Operational Research Society, vol. 49, pp. 237-252, Mar 1998.

[11] D. Rose, R. E. Tarjan, and G. Lueker, "Algorithmic Aspects of Vertex Elimination on Graphs," SIAM Journal, pp. 5:146-160, 1976.

[12] D. R. Fulkerson and O. A. Gross, "Incidence Matrices and Interval Graphs," Pac. J. Math, vol. 15, pp. 835-855, 1965.

[13] K. Sundaresan and R. Sivakumar, "A Unified MAC Layer Framework for Ad Hoc Networks With Smart Antennas," in Technical Report, GNAN Research Group, Mar 2004.

[14] S. Krishnamurthy, A. Acampora, and M. Zorzi, "Polling based Media Access Protocols for Use With Smart Adaptive Array Antennas," in IEEE Trans. on Networking, Apr. 2001.

[15] A. Nasipuri, K. Li, and U. R. Sappidi, "Power Consumption and Throughput in Mobile Ad Hoc Networks Using Directional Antennas," in Proc. of IEEE IC3N, Oct. 2002. 